\title{
ANÁLISIS DE UN PERFIL NUEVO EN TERRAZA DE LAS JARILLAS (PLEISTOCENO MEDIO-SUPERIOR), SEVILLA: YACIMIENTO PIONNER J. A
}

\author{
ANALYSIS OF A NEW STRATIGRAPHIC SECTION IN TERRAZA DE LAS JARILLAS \\ (MIDDLE AND UPPER PLEISTOCENE), SEVILLA. PIONNER J. A. SITE
}

por

\author{
JOSÉ ANTONIO CARO GÓMEZ
}

\section{1.-INTRODUCCIÓN}

Como resultado de las recientes campañas de Prospección Superficial llevadas a cabo durante los dos últimos años en el sector Carmona-Sevilla del Bajo Guadalquivir con motivo de la realización de mi Tesis Doctoral, han sido numerosas las localizaciones y yacimientos paleolíticos encontrados tanto de carácter superficial como en conexión con los depósitos aluviales.

Con el presente trabajo queremos dar a conocer un nuevo perfil situado en uno de los yacimientos más interesantes descubiertos hasta el momento. Este interés se centra en el hecho de hallarse estratigráficamente bien situado y, al mismo tiempo, en la particularidad de encontrarse asociados la industria lítica y los restos paleontológicos.

\section{2.- LOCALIZACIÓN Y CONTEXTO GEOMORFOLÓGICO}

En el valle del Guadalquivir, en el curso de las investigaciones actuales (Díaz del Olmo y otros, 1989; Díaz del Olmo y otros, 1993; Baena, 1993), se han podido reconer hasta 14 niveles fluviales completos en la margen izquierda, entre $+200-210 \mathrm{~m}$. de altura respecto al cauce y la actual llanura aluvial que forman un sistema de terrazas bastante complejo y que se extiende, casi sin interrupción, desde su tramo alto en la provincia de Jaén hasta el estrechamiento del valle al sur de la provincia de Sevilla.

Para su caracterización geomorfológica e interpretación se han agrupado en tres grandes conjuntos:

- Complejo de Terrazas muy altas y altas (T1 a T9)

- Complejo de Terrazas medias (T10 a T12)

- Complejo de Terrazas bajas y muy bajas

En el Bajo Guadalquivir, a partir de la desembocadura del Genil, el conjunto de Terrazas medias que está presente en ambas riberas del río alcanza su máxima extensión en el sector comprendido entre 
Carmona y Sevilla, ya sobre la margen izquierda. Cabe destacar en este sector el alto grado de solapamiento de los niveles y su destacada potencia (más de $15 \mathrm{~m}$.), sobre todo en las cercanías de Sevilla.

Este complejo está compuesto por un conjunto de terrazas entre +55 y $+26 \mathrm{~m}$., de la T10 a la T12, presentes a lo largo de todo el eje fluvial aunque con un desarrollo espacial diferenciado según sectores.

El perfil denominado Pionner J.A. (2a) se encuentra ubicado en el nivel T-12 de este complejo de Terrazas medias, en el límite del mismo en contacto ya con las Terrazas bajas, con una topografía de +30 $\mathrm{m}$.,formando parte de una gran área de explotación de áridos situada hacia el kilómetro 12 de la carretera Sevilla-Brenes.

\section{3.- ANÁLISIS DEL PERFIL PIONNER J.A. (2a)}

\section{1.) SECUENCIA SEDIMENTARIA}

Este perfil, con una potencia de $6 \mathrm{~m}$., se define de muro a techo, por los siguientes niveles:

1.- Depósito de gravas masivas (Gms) compactas, localmente pueden identificarse bolsadas de gravillas imbricadas. El límite inferior no se reconoce, el superior es plano.

Los cantos, de redondeados a subangulosos, son mayoritariamente de cuarcita; la orientación preferente del eje mayor de los mismos es NW-SE. El centilo se situa en torno a los $8,5 \mathrm{~cm}$. Se ha podido constatar la presencia de algún canto blando (nódulo de arcilla) de dimensiones pequeñas.

La potencia total del depósito se situa en torno a los $61 \mathrm{~cm}$.

De este nivel se han extraído restos líticos y óseo que serán analizados posteriormente.

2.- Depósito de arenas finas que entran acuñandose lateralmente, límite plano y neto superior, $(15 \mathrm{~cm}$.)

3.- Depósito limo-arcilloso con gravas, gravillas y arenas, estas últimas más abundantes en la parte superior, $(55 \mathrm{~cm}$.).

Se encuentra muy compactado y entra sobre el nivel inferior de gravas decapitándolo. También se ha detectado la presencia de algunos nódulos alargados de carbonato, de sección circular y $15 \mathrm{~cm}$. de longitud. Límite superior plano y neto.

4.- Depósito de arenas finas compactadas $(15 \mathrm{~cm}$.), similar al nivel 2. Límite superior plano y neto.

5.- Sucesión alternante de fases arenosas y otras limo-arcillosas, con contacto discordante. Los niveles oscilan entre los 8 y los $12 \mathrm{~cm}$. Límite superior plano y neto. Potencia total $72 \mathrm{~cm}$.

6.-Depósito limo-arcilloso $(1,75 \mathrm{~m}$.), pardo-amarillento, con grietas de retracción rellenas de arcilla. Límite superior plano.

7.- Este nivel está compuesto por un fuerte desarrollo edafológico consistente en la superposición de horizontes, de arriba abajo, Ap/Bt/Btca/Bca.

Los horizontes Bt están caracterizados por sus tonos rojos 2,5 YR 4/6, su estructura prismática y descarbonatación del horizonte. Mientras que los carbonatados presentan gruesos núcleos calcáreos muy 
compactos, algunos de tipo septaria, siempre con desarrollo vertical y matriz parduzca. De este horizonte con septarias, pero en otro perfil (Jarillas Martillo; Baena, 1993), han sido extraidas dos muestras para su estudio paleomagnético, aportando en ambos casos polaridad Normal de época Brunhes (aprox. 100.000 - 80.000). También se extrajo una muestra de los carbonatos que arrojó una datación U/Th de 80.000 B.P.

La potencia total del nivel se acerca a los $3 \mathrm{~m}$., el suelo pardo superficial se encuentra decapitado en la casi totalidad del corte.

\section{2.) ANÁLISIS DE LA INDUSTRIA LÍTICA}

Las piezas líticas extraídas en el perfil han sido las siguientes:

1.- Resto nucleiforme de cuarcita de grano fino de color grisáceo claro de muy buena calidad para la talla. Se encuentra totalmente tallado sin restos apreciables de córtex y bastante agotado. El eje mayor mide $4 \mathrm{~cm}$., y el menor $3,5 \mathrm{~cm}$. Las extracciones son pequeñas tendiendo a hacerse de forma centrípeta. No se aprecia rodamiento alguno, sí una pátina clara. ( $\mathrm{N}^{\circ} 2$ del dibujo. Fig. II).

2.- Núcleo irregular de sílex de una sola extracción, aunque parece que hubo otras anteriores; se trata de un canto subanguloso muy poco rodado de color verdoso, muy patinado. Sus dimensiones son $5 \times 4 \mathrm{~cm}$. ( ${ }^{\circ} 3$ del dibujo. Fig. II).

\section{3) INTERPRETACIÓN GEOMORFOLÓGICA, CRONOLÓGICA Y CULTURAL}

Los depósitos de barras de gravas, gravillas y arenas que están en la base del perfil sobremontados por depósitos limosos representan la fase final de esta secuencia aluvial, que se caracteriza por el solapamiento de nuevos ciclos de aluvionamiento sobre los ya existentes.

Los diferentes depósitos limo-arcillosos y arenosos superiores (nivel 5) denotarían una gran llanura de inundación que cíclicamente iba recibiendo los aportes más finos del río en sus crecidas temporales, dando lugar en algunas ocasiones a formaciones, como el nivel 6, de encharcamiento temporal como lo confirma la existencia de grietas de retracción.

Finalmente el perfil queda culminado por un potente desarrollo edafológico en cuya evolución ha interferido el impacto de las crisis morfoclimáticas. En nuestro caso parece corresponderse con la secuencia típica de los valles del dominio mediterráneo con ambientes cálidos y húmedos reflejados, entre otras cosas, en la presencia de fauna como el Elephas Antiquus y el Equus Hydruntinus.

Cronológicamente el perfil Pionner J.A. (2a) queda caracterizado por la correlación paleomagnética realizada en un corte anexo en la base del nivel 7 (horizontes carbonatados) que han aportado una cronología de época Normal Brunhes (100.000-80.000 aprox.).

La secuencia cultural paleolítica establecida para el Bajo Guadalquivir(Vallespí y otros, 1988) atribuye a estos niveles aluviales, depósitos 5 a 1 e iferiores, el desarrollo de un Achelense final transicional que daría paso inmediato al Paleolítico Medio, calificado por Vallespí como Postachelense (Vallespí, 1988, 1989), de los niveles inmediatos y de las formaciones superficiales sobre todo de tipo lacustre y de pequeños encharcamientos, y suelos fersialíticos. 


\section{4.- RELACIONES LOCALES. DISCUSIÓN}

El perfil descrito forma parte de un conjunto de perfiles estudiados en estos niveles aluviales de la T12 a una topografía regular de $+26-29 \mathrm{~m}$., y que ha recibido el nombre genérico de Nivel Jarillas, donde se reune uno de los conjuntos estratigráficos más importantes de todo el valle Medio y Bajo del Guadalquivir. La secuencia de esta localización queda definida por 4 perfiles: Viejas Jarillas, Jarillas Martillo, Pionner Fdo., y Pionner J.A. (Baena, 1993).

Este perfil nuevo que ahora damos a conocer, con una secuencia similar a los mencionados, se desarrolla como hemos visto de techo a muro, hasta el comienzo de los niveles aluviales de gravas de estos depósitos, cuyo desarrollo total no puede dejar de mencionarse ya que la industria lítica se localiza a lo largo de todo el perfil siendo estos depósitos los más importantes desde un punto de vista arqueológico. Por ello es necesario hacer una breve descripción de los restantes niveles hacia muro, tomando como referencia el perfil más próximo (Pionner J.A.), del que forma parte:

Bajo el nivel 1 descrito se encuentra un depósito alternante de arena y gravilla con estratificación cruzada planar sedimentaria y laminaciones de ripples migrando en las secuencias arenosas (Sp y $\mathrm{Sr}$ ). Esta estructura de ripples se encuentra cortada por la entrada de gravilla, con una dirección de la paleocorriente en sentido WNW-ESE. Más abajo, con contacto neto y plano, le sucede un pequeño nivel de unos $5 \mathrm{~cm}$. aproximadamente de gravas muy cementadas con matriz arenosa. Seguidamente un banco de gravas gruesas masivas con barras de cantos y arenas ( $\mathrm{Gms}$ y $\mathrm{Gp}$ ) de unos $3 \mathrm{~m}$. de potencia. Hay predominio de las cuarcitas, acompañadas de sílex, porfidita y pizarras principalmente.El centilo oscila entre los 10 y 14 $\mathrm{cm}$.

Finalmente, bajo el nivel freático, se encuentra el sustrato de margas azules.

A lo largo de todo el Perfil Jarillas los depósitos proporcionan un número considerable de restos arqueológicos y Paleontológicos; la mayoría proceden de los niveles de arenas y gravillas (Sp y $\mathrm{Sr}$ ), también son abundantes en los bancos inferiores de gravas gruesas ( $\mathrm{Gms}$ y $\mathrm{Gp}$ ); en los depósitos superiores de arenas finas (Sp) son más escasos.

En las cercanías del perfil que estamos analizando dentro del yacimiento denominado Pionnner J.A., podemos hablar de la existencia de una industria en conexión de más de 200 piezas compuesta por: 84 núcleos, de los cuales 64 son de cuarcita y 22 de sílex. La mayoría de ellos son irregulares, algunos centrípetos y solamente 2 de técnica levallois. 3 lascas-núcleo, una de ellas tiene una extracción de lasca conweba. 91 lascas ( 73 de cuarcita, 25 de sílex), la mayoría son simples, aunque existe 4 pseudolevallois. 5 cantos tallados todos ellos de talla unifacial. 1 Bifaz parcial de pequeñas dimensiones de cuarcita. 4 picos triédricos de cuarcita. 2 hendedores de cuarcita de tipo 0.3 raederas de cuarcita, una de ellas bifacial. 1 raspador de cuarcita. 3 cuchillos de dorso atípico y 1 cuchillo de dorso natural de cuarcita. 6 muescas, 1 de ellas de sílex. 1 denticulado de cuarcita y 7 diversos de cuarcita.

Atendiendo al rodamiento de todas estas piezas se podrían deferenciar varias series, siendo la más numerosa aquella en la que los restos están frescos o muy poco rodados. La serie más rodada sería la menos importante en cuanto a su número.

Los núcleos suelen ser de grandes dimensiones y con pocas extracciones. Los levallois son pequeños y los centrípetos de mediano tamaño siendo mayoritarios los discoides.

Las lascas suelen ser corticales o semicorticales, las hay de todos los tamaños predominando los medianos, las de sílex son mayoritariamente pequeñas.

En cuarcita casi todos los talones son corticales; en sílex hay algunos lisos.

Todas estas características precisarían unas matizaciones diferentes según los niveles donde aparecen. 


\section{5.- VALORACIONES FINALES. CONCLUSIONES}

Esta industria por las características descritas y por su posición cronoestratigráfica creemos que se corresponde con el Achelense Final Transicional propio del final de la secuencia de las terrazas medias del Bajo Guadalquivir (T-12). Tipológicamente este Achelense final se caracteriza por la reducción del bloque común de cantos tallados, bifaces, hendedores y triedros, y el notable desarrollo en tipos de la lista normativa, modos de retoque; un aumento de los grupos levallois y musteriense, y algo menos del grupo Paleolítico Superior (Vallespí, 1992).

Existe otro conjunto lítico recogido en superficie pero con abundantes restos de matriz, de dimensiones mucho más pequeñas y con un porcentaje de sílex superior, donde los tipos sobre lasca son más numerosos y que tipológicamente podrían incluirse en el denominado Paleolítico Medio postachelense.

En un primer análisis comparativo de la matriz y los depósitos descritos, parece que podrían situarse en el final de la secuencia aluvial sobre las arenas finas (Sp) o al final del nivel 6, limo-arcilloso (Fm), lo que estaría en consonancia con otros yacimientos bien situados estratigráficamente en estos niveles de terrazas medias, y cronológicamente con las dataciones radiométrica y paleomagnética realizadas en éstos.

En los restantes perfiles que caracterizan la serie de Jarillas la industria lítica, aunque más escasa, se corresponde perfectamente con el análisis efectuado para la de Pionner J.A.

Igualmente que la industria lítica también han aparecido en los niveles de gravas y arenas restos paleontológicos que por el momento están en su gran mayoría sin estudiar. De unas primeras observaciones se puede destacar la presencia de Elephas Antiquus, Equus sp., Bos sp., Ciervo sp. y otros restos de vertebrados indeterminados.

En la localización Antiguos Viveros (T12) con una topografía de $+20-31 \mathrm{~m}$. , y con los mismos rasgos generales que caracterizan la serie litoestrotigráfica de las Jarillas, en el nivel de gravas y arenas (Gms y Gp) se obtuvieron restos paleontológicos de Equus Hydruntinus y Elephas Antiquus.

Como hemos podido comprobar el perfil Pionner J.A. se trata de una secuencia completa con pavimientos de barras basales formados por gravas masivas, separados a techo, erosivamente por barras de gravas y lechos de arenas con acentuación de los procesos migratorios de canales, manifestándose un nuevo ciclo de aluvionamiento.

Parece que ahora el factor dominante en la dinámica del Guadalquivir (Baena, 1993), no sería morfoclimático sino que estaría influido por la tectónica regional, condicionando tanto la morfología del valle como los ritmos de sedimentación y erosión que han dado lugar a que la potencia de estas terrazas sea bastante considerable, en nuestro caso puede llegar a los 14 ó $15 \mathrm{~m}$, debido a un fenómeno de subsidencia.

Sobre los aluvionamientos solapados se superponen depósitos limosos con desarrollos de suelos rojos y pardos fersialíticos.

Por todas las características expuesta en la estratigrafía general podemos decir que el yacimiento de Pionner se trata de un depósito aluvial de acreción lateral, es decir, no estaríamos en el centro del canal profundo, aunque los niveles más inferiores con cantos muy gruesos quizás sí lo serían, el resto con gravas y gravillas en formaciones tabulares se encontrarían relativamente apartados del cauce, se conformarían en orillas donde a causa de la baja energía desarrollada por el río irían acumulandose los restos arrastrados por el mismo: huesos de animales, industria lítica, etc., que al presentarse en su inmensa mayoría sin rodar o con un leve rodamiento no deben de haber rocorrido demasiada distancia desde el lugar donde fueron depositados. 


\section{6.- BIBLIOGRAFÍA}

BAENA, R. (1993): «Evolución Cuaternaria (3 M.a.) de la Depresión del Medio-Bajo Guadalquivir y sus Márgenes (Córdoba y Sevilla). Tesis Doctoral inédita.

DÍAZ DEL OLMO, F.; VALLESPÍ, E.; BAENA, R. y RECIO, J.M. (1989): «Terrazas pleistocenas del Guadalquivir occidental: geomorfología, suelos, paleosuelos y secuencia cultural», El Cuaternario en Andalucía Occidental, AEQUA, Monografías, 1, p. 33-42.

DÍAZ DEL OLMO, F.; VALLESPÍ, E. y BAENA, R. (1993): «Proyecto: Formaciones Cuaternarias y secuencia paleolítica en el Bajo Guadalquivir», Investigaciones Arqueológicas en Andalucía, 19851992. Proyectos, p. 193-210. Huelva.

VALLESPÍ, E. (1988): «Paleolítico Medio de aspecto postachelense en la Depresión Inferior del Guadalquivir», Homenaje al Prof. E. Ripoll Perelló; Espacio, Tiempo y Forma, serie I, Prehistoria, T.I. p. 85-91, UNED.

(1989): «Paleolítico Medio indeterminado de aspecto postachelense, en ámbitos fluviales del Sur y Centro de la Península Ibérica», Velia, 6, p. 7-20.

VALLESPÍ, E.; DÍAZ DEL OLMO, F.; ÁLVAREZ, G. y VALLESPÍ GARCÍA, E. (1988): «Secuencia paleolítica del Bajo Guadalquivir», Revista de Arqueología, nº 82, p. 8-17. 


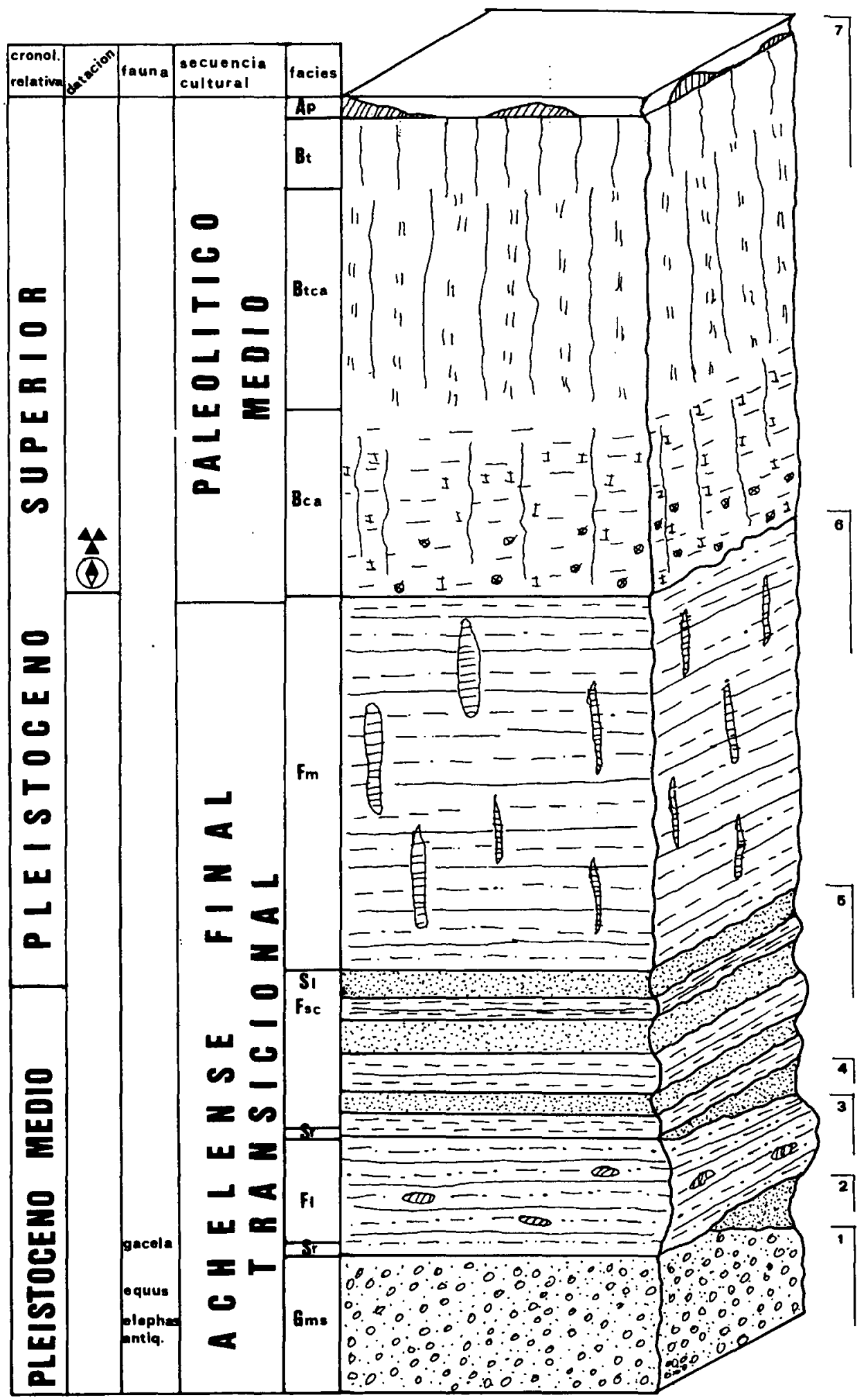

 


\section{FIGURA 2}

(a) Desarrollo detallado de los niveles 1, 2, 3 y 4 .

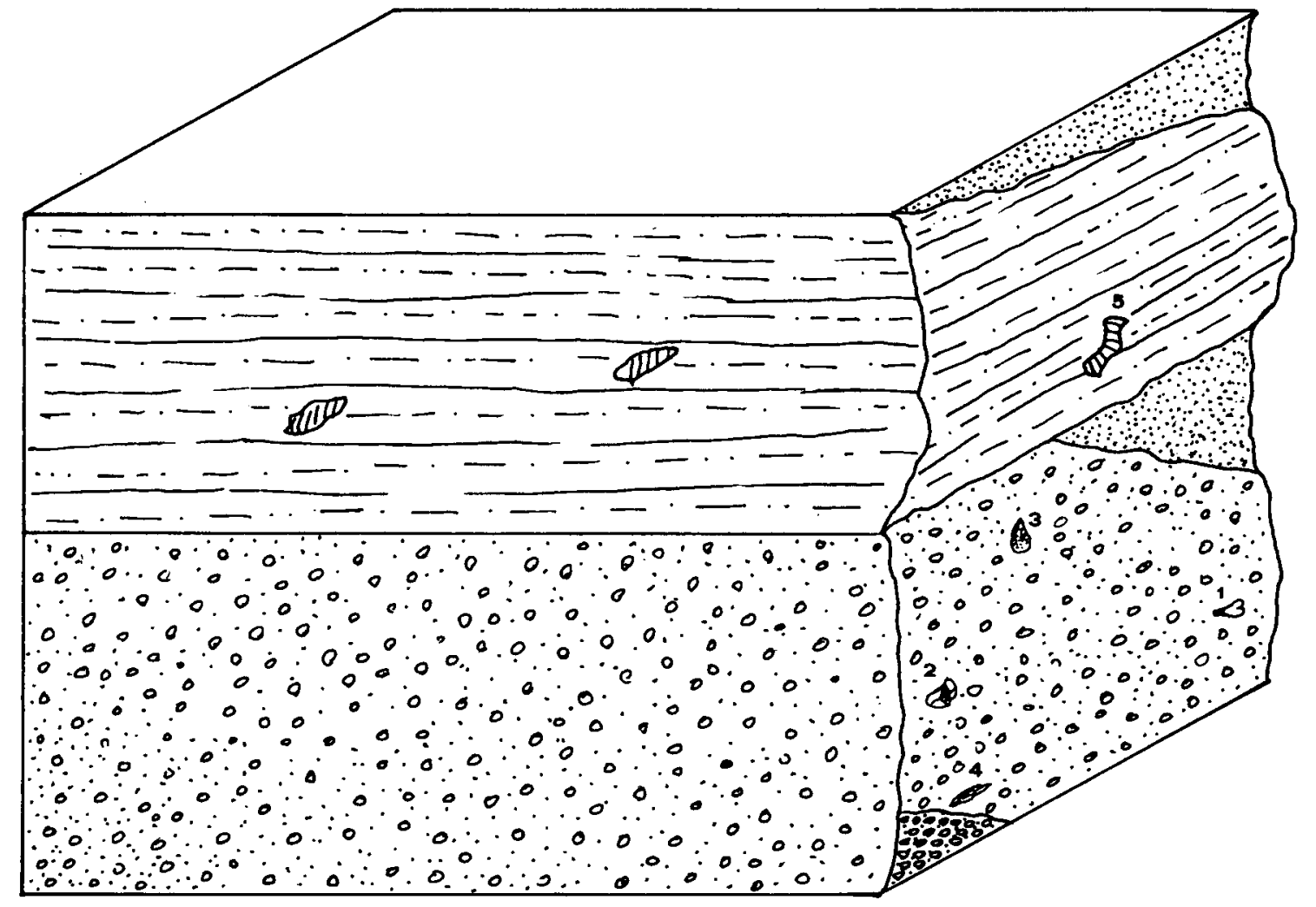

\section{FIGURA 2}

(b) Lectura de los restos indicados:

1.- Resto óseo situado a $18 \mathrm{~cm}$. de la base

2.- Pieza nucleiforme de cuarcita a $30 \mathrm{~cm}$. de la base

3.- Núcleo irregular de sílex situado a $48 \mathrm{~cm}$ de la base

4.- Nódulo de arcilla (canto blando) a $19 \mathrm{~cm}$ de la base

5.- Nódulo de carbonato

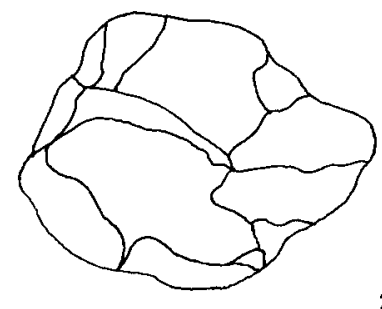

2

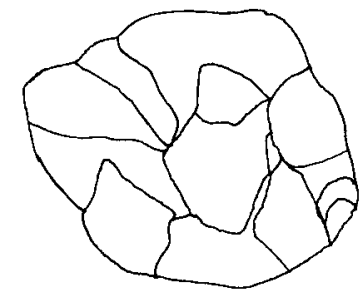

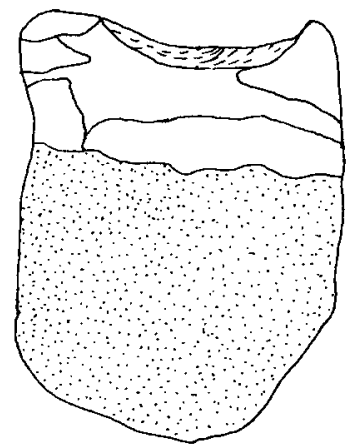

3

ISSN: 1133-4525 ISSN-e: 2255-3924 http://dx.doi.org/10.12795/spal.1993.i2.13 